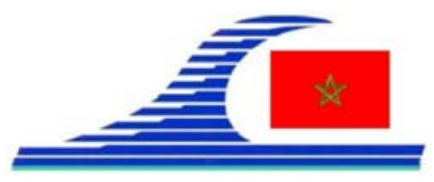

Conférence Méditerranéenne Côtière et Maritime

EDITION 2, TANGER, MAROC (2011)

Coastal and Maritime Mediterranean Conference

Disponible en ligne - http://www.paralia.fr - Available online

\title{
Amélioration du comportement mécanique de sédiments stabilisés au ciment par un renforcement à base de fibres
}

\author{
Yingjie LIANG ${ }^{1}$, Daniel LEVACHER ${ }^{1}$, Nabil CHERIFI ${ }^{1}$
}

1 Université de Caen, Laboratoire de Morphodynamique Continentale et Côtière, M2C UMR 6143, 24 rue des Tilleuls, 14000 Caen, France

yingjie.liang@unicaen.fr ; daniel.levacher@unicaen.fr

\section{Résumé :}

Pour le respect de l'environnement de grands volumes de sédiments marins dragués doivent être valorisés. Usuellement, ils sont stabilisés aux liants hydrauliques donnant lieu à de rares applications routières, leurs performances mécaniques étant insuffisantes. Mais un ajout de fibres-déchets végétales ou artificielles peut modifier, renforcer et améliorer les performances mécaniques de sols fins stabilisés au ciment. Des mélanges composites sédiments-fibres ont été étudiés : effet des dosages et de la taille des fibres. Les indicateurs mécaniques pour évaluer le comportement renforcé de sédiments stabilisés au ciment, sont les résistances en compression $R c$ et en traction indirecte Rt. Les fibres-déchets considérées sont des fibres de lin et des fibres géosynthétiques.

\section{Mots-clés :}

Sédiment marin - stabilisation - ciment - fibres souples - lin - fibre géosynthétique résistance à la compression - traction indirecte - renforcement

\section{Introduction}

Une gestion durable intelligente des sédiments marins peut contribuer à l'économie de ressources en granulats. Encore faut-il en améliorer leurs performances mécaniques? La stabilisation de sédiments à base de liants hydrauliques a été largement étudiée (COLIN, 2003 ; SILITONGA, 2010). Les liants classiques en améliorent le comportement à la compression. Toutefois, il convient de les améliorer globalement comme par exemple par un renforcement à base de fibres (TANG et al., 2007). Afin d'éviter un comportement rigide et fragile à tout matériau résultant d'un procédé de stabilisation par ciment et chaux, des fibres peuvent être ajoutées (BASHA et al., 2005). Un concept innovant consiste à co-valoriser des sédiments et des fibres-déchets. L'étude porte sur l'influence de l'ajout de fibres de lin et géosynthétiques. L'évaluation du renforcement par fibres est faite sur des échantillons confectionnés en laboratoire à partir des indicateurs mécaniques $\mathrm{Rc}$ et $\mathrm{Rt}$, respectivement issus d'essais de résistance à la compression et à la traction indirecte.

\section{Constituants des mélanges composites}

Les sédiments retenus proviennent du port de La Baule-Le Pouliguen (LBLP). Ils ont 
La connaissance de la Mer :

un vecteur du développement durable en Méditerranée

été dragués dans deux bassins B1 et B2. Leurs principales caractéristiques sont données dans le tableau 1. Le ciment Portland utilisé est un CEM II B-M (S-LL) 32,5R CE CP1 NF. Les propriétés des fibres de lin sont connues, on peut rappeler que ce sont des fibres souples très résistantes, très fines mais qui peuvent absorber beaucoup d'eau. Les fibres géosynthétiques, référencées $\mathrm{PP}$ résultent d'un mélange de polypropylène et polyéthylène. Quelques caractéristiques sont énoncées dans le tableau 2.

Tableau 1.Caractéristiques physico-chimiques des sédiments LBLP

\begin{tabular}{|c|c|c|}
\hline Caractérisation & Bassin B1 & Bassin B2 \\
\hline \multicolumn{3}{|l|}{ Nature granulométrique } \\
\hline Fraction sableuse (>63 $\mu m,(\%))$ & 15.6 & 9.4 \\
\hline Fraction silteuse (2 à 63رm),(\%) & 78.9 & 83.6 \\
\hline Fraction argileuse $(<2 \mu \mathrm{m}),(\%)$ & 5.5 & 7 \\
\hline Coefficient d'uniformité Cu & 5.9 & 5.9 \\
\hline Coefficient de courbure Cc & 0.9 & 1.1 \\
\hline \multicolumn{3}{|l|}{ Limites d'Atterberg } \\
\hline Limite de liquidité, $W_{L}(\%)$ & 72 & 89 \\
\hline Limite de plasticité, $W_{P}(\%)$ & 42 & 43 \\
\hline Indice de plasticité, $I_{P}(\%)$ & 30 & 46 \\
\hline Teneur en matière organique, $\mathrm{MO}(\%)$ & 11.5 & 11.6 \\
\hline Teneur en carbonates, (\%) & 14.8 & 14.1 \\
\hline Teneur en eau initiale, $W_{i}(\%)$ & 164 & 192 \\
\hline
\end{tabular}

Tableau 2. Caractéristiques géométriques et mécaniques de la fibre synthétique PP.

\begin{tabular}{ll}
\hline Paramètres & Fibre synthétique PP \\
\hline Couleur & blanche \\
Masse volumique & $0.92 \mathrm{~g} / \mathrm{cm}^{3}$ \\
Diamètre & $1.0 \mathrm{~mm}$ \\
Longueur & $50 \mathrm{~mm}$ \\
Point de fusion & $160^{\circ} \mathrm{C}$ \\
Point d'inflammation & $590^{\circ} \mathrm{C}$ \\
Résistance à la traction & $600 \mathrm{MPa}$ \\
Module de Young & $5 \mathrm{GPa}$ \\
Résistance chimique (alcalins,...) & élevée \\
\hline
\end{tabular}

Si l'on observe le tableau 1, les caractéristiques des sédiments issus des deux bassins sont similaires. Les deux sédiments ont donc été mélangés pour l'ensemble des essais de stabilisation. Des fibres en renforcement sont ajoutées dans les sédiments LBLP stabilisés avec $7 \%$ de ciment. Les dosages en fibres sont de $0.2 \%, 0.4 \%$ et $0.8 \%$ exprimés par rapport à la matière sèche de sédiment. Pour chaque dosage, 4 longueurs de fibres ont été retenues, fonction du diamètre $\mathrm{D}(34 \mathrm{~mm})$ de l'échantillon cylindrique 
confectionné, d'élancement 2. Elles sont respectivement de D/8 (4.25mm), D/4 $(8.5 \mathrm{~mm}), \mathrm{D} / 2(17 \mathrm{~mm})$ et $\mathrm{D}(34 \mathrm{~mm})$. Les performances mécaniques sont déterminées à partir d'essais de résistance à la compression simple $R c_{j}$ à j jours $(7,14,28,60$ et 90jours) et à la traction indirecte $\mathrm{Rt}_{\mathrm{j}}$ (28jours). Cette valeur de $\mathrm{Rt}_{28}$ permet d'obtenir la courbe de résistance en traction directe en fonction du module d'élasticité pour des matériaux traités aux liants hydrauliques en couches routière, selon la norme (NF P 98-232-3, AFNOR, 2001).

\section{Résultats et discussions}

4.1 Effet de l'ajout de fibres sur la résistance en compression $\mathrm{Rc}_{\mathrm{j}}$

Les figures 1 et 2 présentent les évolutions de $\mathrm{Rc}_{\mathrm{j}}$ en fonction des différents dosages et longueurs de fibres utilisées (fibre souple végétale : du lin et fibre semi-rigide géosynthétique PP) pour les sédiments LBLP stabilisés avec $7 \%$ de ciment. Globalement, les valeurs obtenues de $\underline{R c}_{j}$ sont plus élevées avec les fibres souples végétales (lin), figures 1a et 2a. Mais pour les 2 types de fibres mélangées, la valeur optimale de $\underline{\mathrm{RC}_{\mathrm{j}}}$ est toujours obtenue avec un dosage de $0.4 \%$ de fibres de $17 \mathrm{~mm}(\mathrm{D} / 2)$ de longueur.
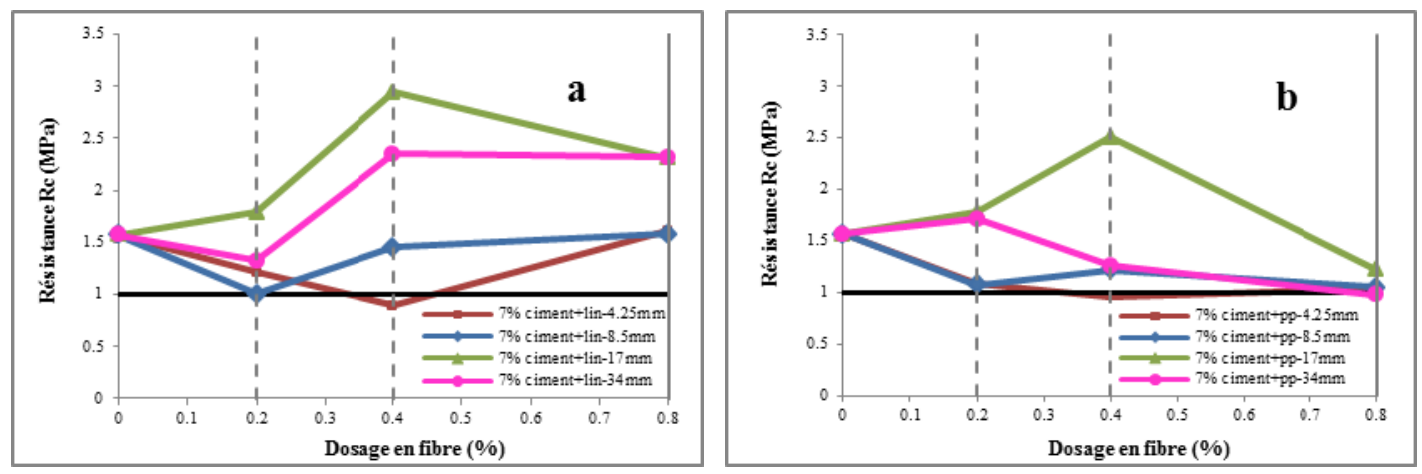

Figure 1. Effet des dosages en fibres sur les valeurs de $R c_{28}$.
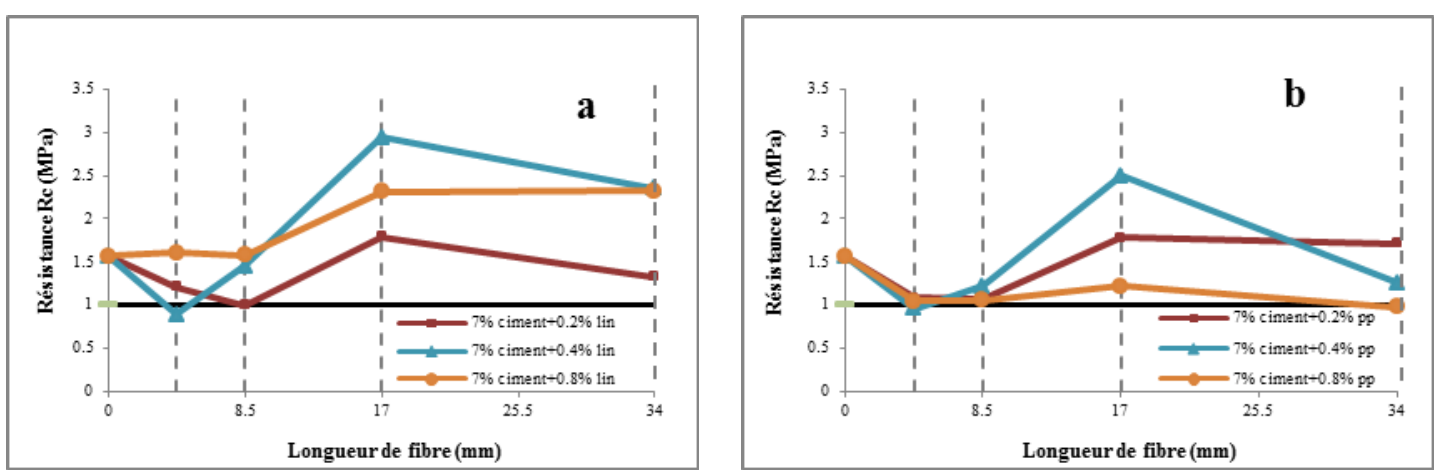

Figure 2. Effet des longueurs de fibres sur les valeurs de $R c_{28}$ pour 3 dosages. 
La connaissance de la Mer :

un vecteur du développement durable en Méditerranée

Les relations contrainte-déformation obtenues à partir des essais de compression simple réalisés sur le sédiment stabilisé à $7 \%$ de ciment, non fibré ( $0 \%$ de fibres) et avec l'ajout des 2 types de fibres de même longueur optimale et dosées à $0.2 \%, 0.4 \%$, et $0.8 \%$, sont illustrées à la figure 3. On constate l'évolution des ruptures. Il y une rupture immédiate type matériau fragile sur l'échantillon non fibré. Un apport de $0.8 \%$ de fibres de lin et géosynthétiques, confère un comportement ductile au sédiment stabilisé. La figure 3b montre une valeur de $\mathrm{Rc}_{28}$ pour un dosage de $0.8 \%$ de fibres géosynthétiques (fibres semi rigides) plus faible que celle obtenue pour le sédiment non fibré.
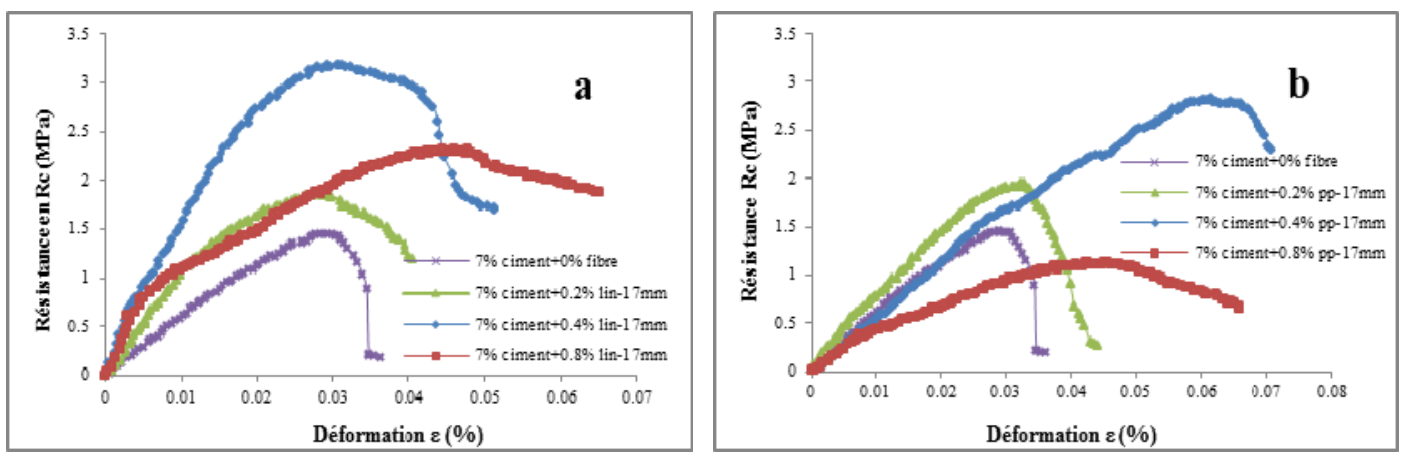

Figure 3. Comportement à la compression en contrainte-déformation des éprouvettes non fibrées et renforcées à 28 jours.

\subsection{Effet de l'ajout de fibres sur la résistance en traction indirecte $\mathrm{Rt}_{\mathrm{i}}$}

Les relations contrainte-déformation obtenues à partir des essais en traction indirecte réalisés sur le sédiment stabilisé à $7 \%$ de ciment, non fibré et un ajout des 2 fibres de même longueur optimale et dosées à $0.2 \%, 0.4 \%$, et $0.8 \%$, sont données à la figure 4 . La valeur de $\mathrm{Rt}_{28}$ a tendance à croître avec le dosage en fibres souple de lin (figure 4a), mais en revanche, la tendance s'inverse pour le sédiment stabilisé avec des fibres géosynthétiques qui sont plus rigides.
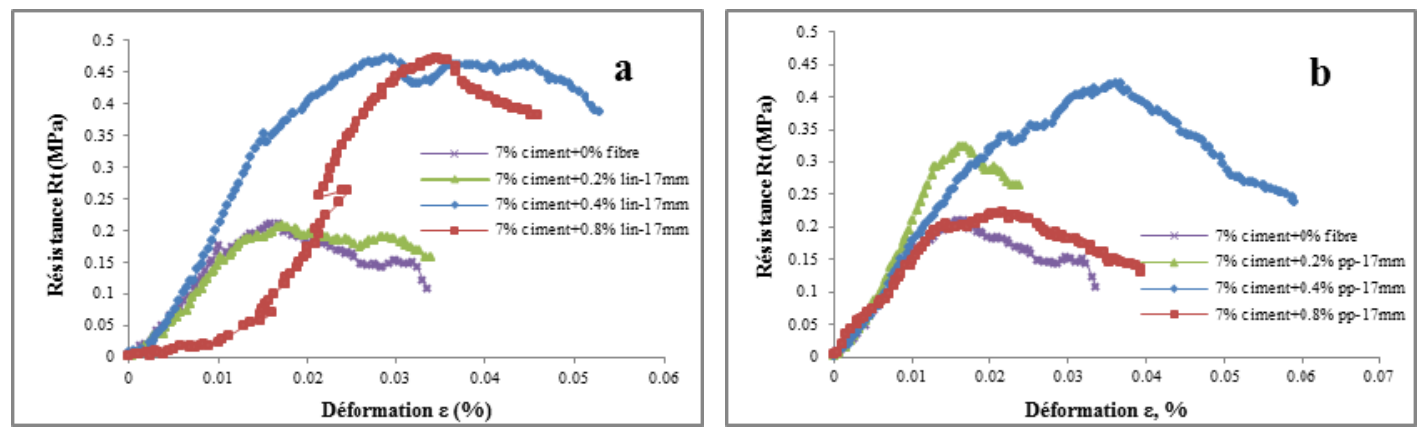

Figure 4. Comportement à la traction indirecte en contrainte-déformation des éprouvettes non fibrées et renforcées à 28 jours. 
La figure 5 montre la relation établie entre $\mathrm{Rt}_{\mathrm{j}}$ en traction directe et $\mathrm{E}$, pour le sédiment stabilisé avec $7 \%$ de ciment et $0.4 \%$ de fibres de $17 \mathrm{~mm}$. Le matériau fibré est classé en S1, sans fibre, il appartient à la classe S2 et/ou S3, (NF EN 14227-3, AFNOR, 2004).

\section{Conclusion}

Un ajout de fibres dans un sédiment stabilisé au ciment à un taux faible peut modifier la performance mécanique du matériau résultant. Les valeurs maximales de Rc et Rt sont obtenues pour un dosage de $0.4 \%$ de fibres de lin et/ou de fibres géosynthétiques de longueur égale à la moitié du diamètre de l'éprouvette. Les fibres de lin sont plus efficaces pour la valorisation des sédiments grâce à leurs propriétés intrinsèques dont la souplesse de ces fibres. Par ailleurs les fibres de lin constituent un apport intéressant du point de vue économique (fibres-déchets) et du point de vue environnemental (fibres végétales).

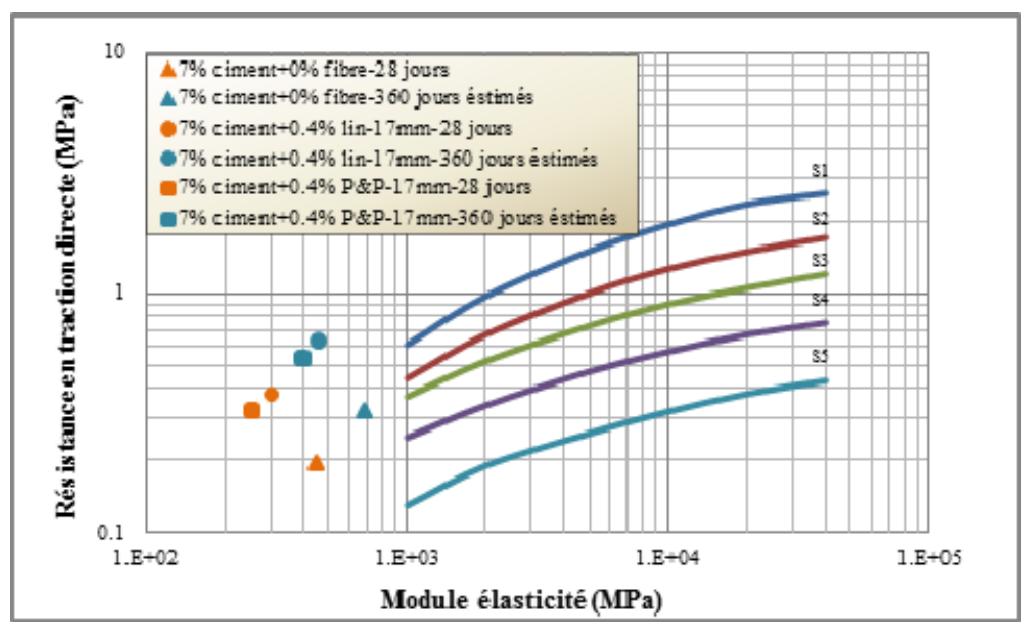

Figure 5. Evolution de la performance mécanique des sédiments stabilisés.

L'évolution à long terme des caractéristiques mécaniques des mélanges à base de fibres n’a pas été abordée dans cette étude. Si les fibres synthétiques, composants de la plupart des géotextiles du génie civil, ont des performances à long terme bien déterminées, ce n’est pas le cas pour les fibres de lin. Cependant dans le concept «sédiment-liant pouzzolanique-fibres végétales », il est supposé que s’il y a dégradation éventuelle des fibres dans le temps, l'effet des liants pouzzolaniques se traduira par une augmentation des résistances à long terme qui viendra pallier en partie cette dégradation. Des essais de durabilité et de résistances aux actions climatiques voire chimiques, sont à envisager pour valider la pérennité de ces mélanges. D’ailleurs des résultats de travaux ont déjà publiés sur cette problématique relative au comportement mécanique de mélanges à base de fibres (GHAVAMI et al., 1999 ; CONSOLI et al., 2002 ; CAI et al., 2006). 
La connaissance de la Mer :

un vecteur du développement durable en Méditerranée

\section{Références bibliographiques}

AFNOR (2001). NF P 98-232-3 : Détermination des caractéristiques mécaniques des matériaux traités aux liants hydrauliques. 11 p.

AFNOR (2004). NF EN 14227-3: Mélanges traités aux liants hydrauliques Spécifications - Partie 3 : Mélanges granulaires traités à la cendre volante.

BASHA E.A., HASHIM R., MAHMUD H.B., MUNTOBAR A.S. (2005). Stabilization of residual soil with rice husk ash and cement. Construction and Building Materials 19 (6), pp 448-453.

CAI Y., SHI B., CHARLES W.W., TANG C. (2006). Effect of polypropylene fibre and lime admixture on engineering properties of clayey soil. Engineering Geology 87, pp 230-240. doi:10.1016/j.enggeo.2006.07.007

COLIN D. (2003). Valorisation de sédiments fins de dragage en technique routière. Thèse de Doctorat, Université de Caen, 180 p.

CONSOLI N.C., MONTARDO J.P., PRIETTO P.D.M., PASA G.S. (2002). Engineering behaviour of a sand reinforced with plastic waste. Journal of Geotechnical and Geoenvironmental Engineering, ASCE, Volume 128, n 6, pp 462-472.

GHAVAMI K., TOLEDO FILHO R.D., BARBOSA N.P. (1999). Behaviour of composite soil reinforced with natural fibres. Cement and Concrete Composites 21 pp 39-48. doi:10.1016/S0958-9465(98)00033-X

SILITONGA E. (2010). Valorisation des sédiments marins contaminés par solidification/stabilisation à base de liants hydrauliques et fume de silice. Thèse de Doctorat, Université de Caen, 227 p.

TANG B., GAO W., CHEN F., CAI Y. (2007). Strength and mechanical behavior of short polypropylene fiber reinforced and cement stabilized clayey soil. Geotexiles and Geomembranes 25, pp 194-202. doi:10.1016/j.geotexmem.2006.11.002 